\title{
Fatal haemoptysis in a case of lymphomatoid granulomatosis treated with rituximab
}

\author{
S. Jaffre*, F. Jardin\#, S. Dominique*, E. Duet*, Ph. Hubscher ${ }^{\star}$, A. Genevois ${ }^{+}$, \\ F. Corne*, S. Bota*, G. Nouvet* and L. Thiberville*
}

ABSTRACT: Lymphomatoid granulomatosis is a rare angiocentric and angiodestructive disease, which commonly involves the lungs but also the brain, kidneys, liver and skin. This report describes the case of a 33-yr-old female with an aggressive form of lymphoid granulomatosis treated with an anti-CD20 antibody. Dramatic radiological improvement was seen at the fourth week. However, the patient died at home 1 month after the last rituximab administration from a massive haemoptysis.

KEYWORDS: Haemoptysis, lymphomatoid granulomatosis, rituximab

ymphomatoid granulomatosis (LYG), also known as angiocentric lymphoma, is a multisystemic angiocentric lymphoproliferative disease, which predominantly involves the lungs but also other extrathoracic sites such as the upper respiratory tract, skin, kidneys, and peripheral and central nervous systems [1]. LYG usually presents as multiple pulmonary nodules on chest radiograph with rapid progression and excavation. Clinically, the most frequent signs are cough, dyspnoea and chest pain. Fever, malaise and weight loss are also commonly observed. The disease affects adults in middle age, with a higher percentage of males affected than females [2].

Treatment is currently not well established. Spontaneous remission has been reported [3]. In most of the published series, treatment consists of corticosteroid therapy, alone or combined with cyclophosphamide, and produces prolonged complete remission in nearly $50 \%$ of the cases $[3,4]$. In patients not responding to first line therapy or presenting with more aggressive disease, chemotherapy regimen associated with cyclophosphamide, doxorubicin, vincristine and prednisone $(\mathrm{CHOP})$ is frequently proposed [5-7], with complete remission in some cases [8]. The overall prognosis of LYG is poor, with a 5-yr survival rate in $<50 \%$ of cases [3].

This study presents the case of a 33-yr-old female with LYG resistant to high-dose corticosteroids and cyclophosphamide, whose lung lesions dramatically responded to rituximab. The patient, however, later died from massive haemoptysis 1 month after completion of treatment.

\section{CASE REPORT}

A 33-yr-old female was hospitalised with a 3month history of cough, dyspnoea, fever and skin rash. These symptoms progressed despite a course of antibiotics prescribed for presumed community-acquired pneumonia a few weeks prior to admission. The patient's respiratory condition transiently improved with the use of corticosteroids (prednisolone $20 \mathrm{mg} \cdot \mathrm{day}^{-1}$ ), but symptoms reappeared shortly after the corticosteroids were stopped, resulting in hospitalisation.

On admission, the patient had a temperature of $38.5^{\circ} \mathrm{C}$, a heart rate of 110 beats $\cdot \mathrm{min}^{-1}$, and a blood pressure of $110 / 60 \mathrm{mmHg}$. Respiratory crackles were heard over both lung fields. A white blood cell count showed lymphopenia $\left(0.9 \mathrm{~g} \cdot \mathrm{L}^{-1}\right)$, hypogammaglobulinaemia $\left(4.9 \mathrm{~g} \cdot \mathrm{L}^{-1}\right)$ and a low immunoglobulin $\mathrm{G}\left(5.3 \mathrm{~g} \cdot \mathrm{L}^{-1}\right)$. Arterial blood gas levels while breathing room air showed a $\mathrm{pH}$ of 7.44, a carbon dioxide tension of $4.5 \mathrm{kPa}$, and a partial pressure of oxygen of $8.8 \mathrm{kPa}$. A chest radiograph revealed bilateral nodules of variable sizes predominantly in the lower lung fields, and a computed tomography (CT) scan of the thorax showed multiple small excavated pulmonary nodules with poorly defined margins (fig. 1). Fibreoptic bronchoscopy was normal. Bacteriological samples were

\section{AFFILIATIONS}

${ }^{*}$ Clinique pneumologique and +Service de radiologie, CHU Rouen, and

${ }^{\#}$ Service hématologie, Centre Bequerel, Rouen, and

"Service de pneumologie, Hopital Jacques Monod, Le Havre, France.

CORRESPONDENCE

$S$. Jaffré

Service de Pneumologie

Hôpital Guillaume et René Laennec

Boulevard Jacques Monod

44093 Nantes Cedex 1

France

Fax: 33240165241

E-mail: sandrine.jaffre@chu-

nantes.fr

Received:

July 212004

Accepted after revision:

August 152005 

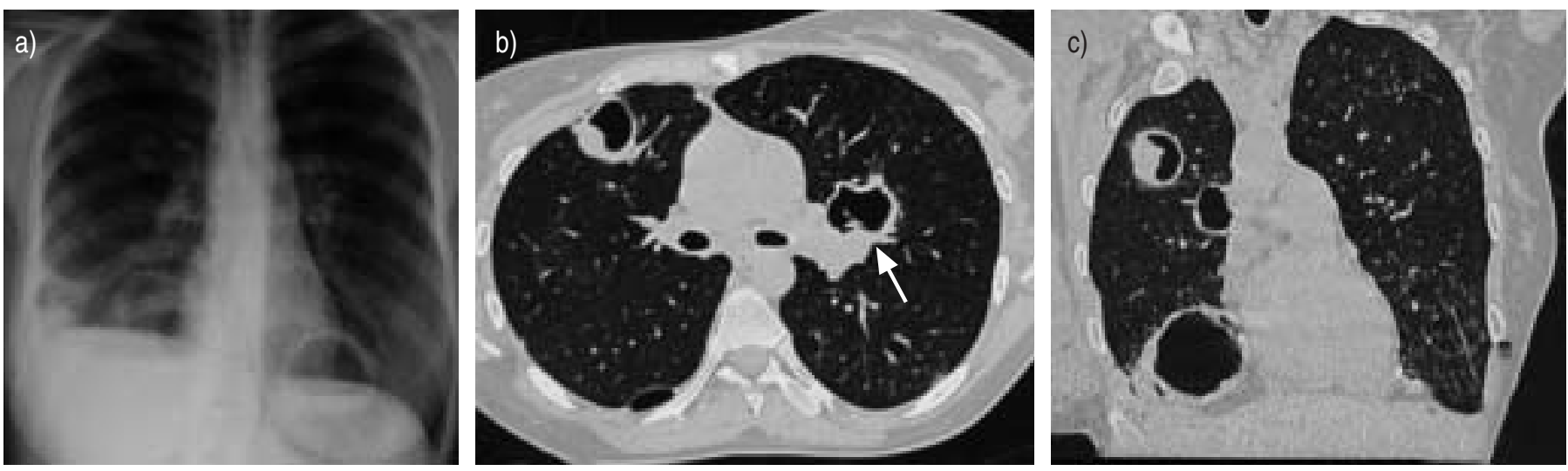

FIGURE 1. A chest radiograph (a) and computed tomography scans ( $b$ and $c$ ) of the thorax before rituximab treatment. A large excavated nodule can be seen adjacent to the left pulmonary artery (indicated by the arrow in b).

negative. Bronchoalveolar lavage showed hypercellularity $\left(0.93 \mathrm{~g} \cdot \mathrm{L}^{-1}\right)$ with $91 \%$ lymphocytes and a CD4/CD8 ratio of 8 .

An open lung biopsy was performed. The pathology analysis was typical of lymphomatoid granulomatosis. Immunohistochemical analysis revealed a predominantly T-cell infiltrate. There were also clusters of large and atypical lymphocytes that stained for CD20 and CD30 B-cell markers $(10 \%)$. Latent membrane protein 1 expression was negative in tumour cells.

High-dose prednisolone $\left(1.5 \mathrm{mg} \cdot \mathrm{kg}^{-1} \cdot \mathrm{day}^{-1}\right)$ was reintroduced and maintained during 6 weeks with slight clinical improvement, but no modification of chest CT scan. A bolus of $750 \mathrm{mg} \cdot \mathrm{m}^{-2}$ cyclophosphamide was administered, but clinical condition worsened, including weight loss, fever and abundant purulent expectoration. The chest radiograph showed nodule progression with hydro-aeric levels. Blood cultures, as well as bacteriological and fungal sputum analyses, were negative. Antibiotherapy using cefotaxim, metronidazol, sulfamethoxazole, trimethoprime and fluconazol was started without success. Due to the resistant nature of the disease and the progression of lung excavation with fever, $375 \mathrm{mg} \cdot \mathrm{m}^{-2}$ of rituximab was administered as a single therapy, once a week for 4 consecutive weeks. Treatment was well tolerated and resulted in immediate apyrexia and improvement in general state of health. Dramatic radiological improvement was seen in the fourth week on a CT scan (fig. 2). However, the patient died at home 1 month after the last rituximab administration from a massive haemoptysis. No autopsy could be performed.

\section{DISCUSSION}

Whilst it was initially classified as a benign disease characterised by pulmonary angiitis and granulomatosis [6], LYG is now considered to be a "T-cell rich" B-cell lymphoma [9]. The diagnosis of LYG is based on histological examination of surgical samples. On histology, the most distinctive feature is the multicellular lymphoid infiltrate, which is composed of small lymphocytes, plasma cells and large, atypical mononuclear cells. In the case reported, clinical and radiological findings were very suggestive of LYG, but the diagnosis was established on open lung biopsy. Dissected lymph node demonstrated a nodular perivascular infiltrate with angiitis and granulomatosis, a triad specific to LYG [1]. The presence of polymorphic lymphocytic cells with occasional large atypical cells expressing B-cell (CD20) antigen confirmed the diagnosis [4].

After using corticosteroid and cyclophosphamide and because of the progression of the lung disease, with lung abcedation and fever despite large spectrum antibiotic and antifungal therapy, rituximab was introduced alone. Rituximab is a chimeric mouse/human monoclonal antibody that specifically targets the CD20 antigen on the surface of normal and malignant human B-cells. This antibody is currently used, frenquently in combination with chemotherapy, in a wide spectrum of B-cell lymphomas, including indolent or aggressive diseases, in both first line and relapse therapy [10].

In the present case, the use of rituximab was mainly based on three considerations. First, it is now clearly demonstrated that the neoplastic LYG clone arises from B-lymphocytes. Recently,
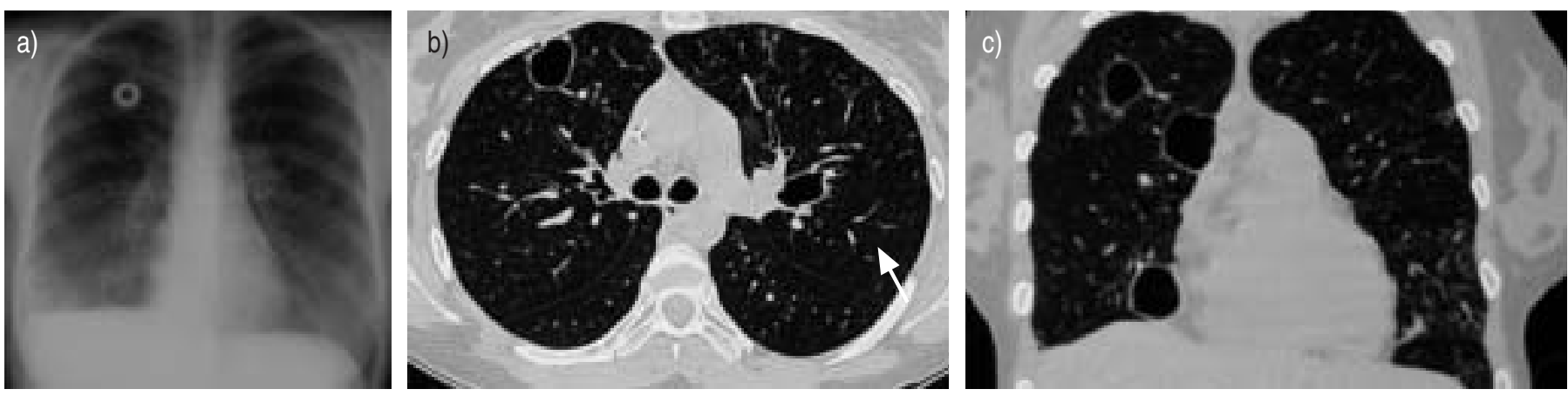

FIGURE 2. A chest radiograph (a) and computed tomography scans ( $b$ and $c$ ) of the thorax 4 weeks after the completion of rituximab treatment. 
rituximab was successfully used in a case of cutaneous LYG occurring in a child with Wiskott-Aldrich syndrome, leading to a persistent remission after 18 months' follow-up [11]. Secondly, rituximab has been successfully used to treat B-lymphoproliferative disorders arising in various immunodeficiency states, including organ and bone marrow transplantations, HIV infection or inherited immunodeficiency. Thirdly, Wegener's granulomatosis, which can also present with excavated lung nodules, has been successfully treated by rituximab after corticosteroids or cyclophosphamide inefficacy, suggesting a significant diffusion of the antibody in the lung and granulomas [12].

In the present report, the use of rituximab as a single therapeutic agent, despite the fact that LYG is generally considered as an aggressive lymphoma, was justified by the lack of severe adverse effect (particularly myelotoxicity) of this agent, reducing the risk of severe sepsis in an infected and highly immunocompromised patient.

The dramatic regression of the lung lesions and the rapid improvement of the general health of the patient in this report, confirms the high activity of rituximab against chemoresistant and aggressive LYG.

The massive haemoptysis that led to the death of the patient should, however, be discussed in relation to this new treatment. Fatal haemoptyses have been described in lung cancer patients treated with bevacizumab, a monoclonal antibody targeting the vascular epidermal growth factor receptor [13]. However, in the present case, rituximab does not appear to be directely involved in the pathogenesis of the haemoptysis, because of the lack of CD20 antigen expression on the surface of the endothelial cells. As an angiocentric and angiodestructive disease, the spontaneous evolution of LYG is often complicated by haemoptysis, leading to death in $40 \%$ of the cases in some series [3]. In the present case, one of the excavated nodules was clearly located near the left pulmonary artery, and may be the cause of the massive bleeding (fig. 1, indicated by the arrow). It cannot be excluded, however, that the rapidity of the rituximab-induced lymphoid tissue regression may have caused the blood vessel wall to become fragile.

In conclusion, rituximab appears to be highly effective in aggressive lymphomatoid granulomatosis. Further studies are required to evaluate the place of rituximab therapy in the treatment of lymphomatoid granulomatosis. These studies should also focus on the frequency and severity of haemoptysis, especially in cases of excavated nodules situated close to the large lung blood vessels.

\section{ACKNOWLEDGEMENTS}

The authors would like to thank R. Medeiros for his advice in editing the manuscript.

\section{REFERENCES}

1 Liebow AA, Carrington CR, Friedman PJ. Lymphomatoid granulomatosis. Hum Pathol 1972; 3: 457-558.

2 Case records of the Massachusetts General Hospital. Weekly clinicopathological exercises. Case 20-1997. A 74year-old man with progressive cough, dyspnea, and pleural thickening. N Engl J Med 1997; 26: 1895-1903.

3 Cadranel J, Wisley M, Antoine M. Primary pulmonary lymphoma. Eur Respir J 2002; 20: 750-762.

4 Jaffe ES, Wilson WH. Lymphomatoid granulomatosis: Pathogenesis, pathology and clinical implications. Cancer Surv 1997; 30: 233-248.

5 Tanière Ph, Thivolet-Béjui F, Vitrey D, et al. Lymphomatoid granulomatosis- a report on four cases: evidence for Bphenotype of tumoral cells. Eur Respir J 1998; 12: 102-106.

6 Summer R, Zacks J, Leong M, O’Regan A. Cough, fever and weight loss in a young male. Eur Respir J 2002; 19: 1210-1213.

7 Lemieux J, Bernier V, Martel N, Delage R. Autologous hematopoietic stem cell transplantation for refractory lymphomatoid granulomatosis. Hematology 2002; 7: 355-358.

8 Donnelly TJ, Tuder RM, Vendegna TR. A 48-year-old woman with peripheral neuropathy, hypercalcemia, and pulmonary infiltrates. Chest 1998; 114: 1205-1209.

9 Lymphomatoid granulomatosis. In: Travis WD, Colby TV, Corrin B, Shimosato Y, Brambilla E. Histological Typing of Lung and Pleural Tumours. Third Edn. Berlin, Springer, 1999: pp. 59-60.

10 Smith MR. Rituximab (monoclonal anti-CD20 antibody): mechanisms of action and resistance. Oncogene 2003; 20: 7359-7368.

11 Sebire NJ, Haselden S, Malone M, Davies EG, Ramsay AD. Isolated EBV lymphoproliferative disease in a child with Wiskott-Aldrich syndrome manifesting as cutaneous lymphoid granulomatosis and responsive to anti-CD20 immunotherapy. J Clin Pathol 2003; 56: 555-557.

12 Specks U, Fervenza FC, McDonald TJ, Hogan MC. Response of Wegener's granulomatosis to anti-CD20 chimeric monoclonal antibody therapy. Arthritis Rheum 2001; 44: 2836-2840.

13 Langmuir V, Cobleigh M, Herbst R, Holmgren E, Hurwitz H, Kabbinavar F. Successful long-term therapy with Bevacizumab (Avastin) in solid tumors. Proc Am Soc Clin Oncol 2002; 21: 9a. 